\title{
Mechanism of Nanning Flood Dike Slope Failure Based on Improved Genetic Algorithm Inversion Analysis
}

\author{
Rongyong $\mathrm{Ma}^{1,}$, , Xiaoqing Zhang, and Ruyue Pang ${ }^{\mathrm{b}}$ \\ ${ }^{1}$ College of Civil Engineering and Architecture, Guangxi University, Nanning, China, 530004 \\ aemail: mry57@gxu.edu.cn \\ bemail: 18775324085@163.com
}

\begin{abstract}
Keywords: dike slope stability; inversion analysis; improved genetic algorithm; strength reduction Abstract. A novel method is established to analyze the dike slope failure mechanism research in this paper. First of all, it improves a serial of operations, and boundary conditions for the genetic algorithm, such as the crossover operation, mutation operation, the operation and the convergence condition, then verifies the global search capability of the improved genetic algorithm with a complex function. Secondly, considering a calculation conditions with falling water levels, combined with the numerical analysis software FLAC3D strength reduction method, the critical state of Nanning city flood control embankment slope near the water surface is inverse analyzed when the water level drops. Finally, an analysis of the flood control dike typical slope failure mechanism under critical condition is also made. The results show that, the inversion analysis method of improved genetic algorithm combined with strength reduction FEM numerical analysis software FLAC3D is extremely effect, which has a powerful ability of global search. It's also fast convergence speed, high accuracy and adaptability.
\end{abstract}

\section{Introduction}

The water level decline is a major reason of breakwater's side slope landslide. Considering the influence of water level changes in flood period to the stability of river breakwater's side slope, and breakwater's side slope infiltration and groundwater infiltration. ChongWu Ma [1] et al. found that there is a critical water level when the river water level rises or falls through numerical calculation and analysis, namely the water level corresponding to the breakwater slope stability coefficient $\mathrm{k}=1.0$. Xinxi Liu [2] et al. analyses the effect of different rainfall conditions of slope stability in the Three Gorges Reservoir area, and they propose that the influencing degree of decreased water level on the stability of landslide in reservoir area depends on the permeability of soil landslide and landslide structure itself. Haiwei Yang [3] uses the finite difference method to simulate the slope landslide in a reservoir area, and analyses the stability of the landslide under different conditions. Weimin Shi [4] researched the influence of the drawdown, permeability to the slope stability. This paper is based on improved genetic algorithm, combined with the strength reduction method of numerical analysis software FLAC3D , and inversion analyze the stability and failure mechanism of breakwater slope in Nanning city.

\section{The inversion method based on improved genetic algorithm}

\section{The standard genetic algorithm}

Genetic algorithm is a kind of new optimization method, using the selective advantage and eliminate disadvantage, namely to imitate the principle of genetic engineering. As a powerful intelligent optimization technique, genetic algorithm has been widely used in each branch of all engineering disciplines. It has the advantage of more in-depth, more easy to use than traditional optimization method in solving global search, design objective function of the complex domain, and complicated target function. It provides a general framework in solving complex optimization problem, and does not depend on the area of regional problems.

Genetic algorithm (GA) still has some shortcomings: a. when it is used to solve some practical problems, GA usually converge to a local solution, not to the global optimal solution of the best. Some 
of them are brought by the objective function, and others were brought by the algorithm itself; $b$. select operation function and intersection weakened gradually with the increase of evolution; c. when a large amount of calculation, the genetic algorithm is slow; d. genetic algorithm has not been effective quantitative analysis method on the precision of the algorithm, credibility, computational complexity, etc. Therefore, we'd better improve on the genetic algorithm to taking more advantage.

\section{The improved genetic algorithm}

The advantages of standard genetic algorithm is easy to implement and well used. But it is very easy to "premature" because of bad robustness of parameter choice. In addition, the convergence speed in the later is slow. This paper put forward a new improved genetic algorithm mainly from genetic crossover and mutation operation two aspects, and proved that the improved algorithm is effective through the example analysis. In this paper, the improved crossover operation and mutation operation, as well as the selection of coding method, the selection operation, the convergence condition is as follows:

(1) Improved method

Crossover operation: a. In order to avoid the relative transmission and maintenance of the offspring of discrete degree, we need to control two parent weighted average of the distance, only has the largest fitness of two individuals is retained; $b$. On the basis of principle a, cross position will change. In the initial stages of evolution, selecting high gene as cross location can search a bigger solution space, to maintain the population genetic differences. In the late stage of evolution, the choice low genetic parent crossover position as individuals is the solution of the optimal solution; c. The application of adaptive crossover probability is important. From the point of the overall development of the individual, crossover probability will be weakened by evolution, ultimately become a stable value. From the perspective of creating new individual genes, all individuals in the group will have equal cross position. Therefore, the genetic algorithm will have a consistent crossover probability in the solution space with all directions. Crossover probability and develop relationship evolution see below type:

$$
P_{c}=\frac{1}{[1+\exp (a \times g)]}+b .
$$

In this formula, $g$ represents the current number of evolution, while a and $\mathrm{b}$ are two constants $(0,1)$ interval value. The test parameters of continuous $b$ value is too large, the algorithm convergence speed, but also very easy to fall into local solution. Algorithm is not stable. If the value is too small, convergence speed will be too slow. Therefore, $b$ takes 0.8 through this article the debugging. In order to meet the change of the early stage of the evolution and the crossover probability, the stability of the algorithm is ensured, and the jump phenomenon is not occurred. Finally, the $a$ takes 0.2.

Mutation operation: Variability is generally considered a backstage operator to ensure that the probability of a special sub-space research problem space is not 0 . Variability has prevented local optimum convergence of the role. In this paper, in the initial stage of the evolution, adaptive mutation probability large differences between individuals, mutation probability should be small. In the later stage of evolution, the differences between individuals is small, it is difficult to produce new individuals, which makes the algorithm converges to local optimal solution. So we need to increase the probability of mutation in the late evolution. At this point the disadvantage individuals will have a greater probability of mutation, and the probability of individual variation is smaller. The probability of variation of individuals in the late stage of evolution can be expressed as:

$$
P_{m}= \begin{cases}\frac{c\left(f_{\max }-f_{j}\right)}{f_{\max }-\bar{f}} ; & f_{j} \geq \bar{f} \\ c & f_{j}<\bar{f}\end{cases}
$$


In this formula, $f_{\max }$ indicates the parent's biggest adaptive value in the process of evolution, $\bar{f}$ indicates the evolution process of individual average fitness value, $f_{j}$ expresses the need for the fitness of individual variation. $C$ is a constants which takes $(0,1)$ interval value. In this paper, a numerical example is analyzed to determine when the value of the $C$ parameter is 0.15 , the algorithm has the best efficiency of cable collection, can effectively avoid falling into the local optimal solution, and avoid premature convergence.

(2) Coding method, selection operation and convergence method

Coding method: The convergence function of genetic algorithm is closely related to the encoding. As a result, the coding method is the primary task of the algorithm. Binary coding is very easy to be used, but must be coded and decoded, which reduces the computational efficiency, and its accuracy is not high. In addition, the string cannot be too long or too short, the use of binary code, if the string is too long, the solution space will be too large, too long, too long, if the string is too short, and the accuracy is not high. Real coding does not need to encode and decode, which improves the accuracy of the algorithm. It can solve the problem of binary code in the face of the cliff, easy to search for a larger solution space. Many experiments show that the convergence speed and the convergence precision of real number coding are better than that of binary coding. Based on the above reasons, this paper applies the real number coding.

Select operation: First, according to the size of the fitness value of the individual, we will have the common nature of the individual into a group. Then, use the disk; select $\mathrm{N}$ times in the group set. Each of the selected groups, as a new individual, is created. In addition, if the maximum adaptation value of the new sub generation is smaller than the maximum of the parent generation, the individual with the maximum fitness value will be copied to the new offspring. Its advantage is to ensure that the algorithm is terminated; the final result is the highest degree of adaptation of the individual.

Convergence conditions: The algorithm has three convergence conditions. If any one of them is satisfied, the algorithm will be stopped. The first is: the largest evolutionary algebra, the second is: convergence precision, third is: if a series of evolution, no new optimal individual, algorithm to stop.

(3) The calculation steps of the improved genetic algorithm

(1) To determine the control parameters: Group $M$ initialization and the maximum number of $G$ evolution;

(2) When $g=0$, given the random initial population with $N$ individuals $\left(x_{1}, x_{2}, \ldots, x_{\mathrm{n}}\right)$;

(3) Selecting the operation of the application of the proposed calculation of fitness value of each individual;

(4) According to the method presented in the paper, do the crossover operation of individual;

(5) According to the method presented in the paper, do the mutation operation of individual $g=g+1$;

(6) If the conditions meet the convergence, stop counting, or go back to step(3).

\section{Example verification}

The standard genetic algorithm (SGA) and the improved genetic algorithm (IGA) is compared in this paper to check the improved genetic algorithm convergence and robustness. We test the performance of the two algorithms by $f_{1}$ function which is widely used, multiple local extreme points and binary multistage peak values. The test functions are as follows:

$$
f_{1}(x, y)=-0.5+\frac{\sin ^{2} \sqrt{\left(x^{2}+y^{2}\right)}-0.5}{\left[1+0.001\left(x^{2}+y^{2}\right)\right]^{2}}, \quad-100<x, y<100
$$

Function $f_{1}$ is a non-quadratic function with two parameters, single extreme. $f_{1}$ has countless extreme points, of which only a $(0,0)$ is a global minimum for -1 . Around the function has a circle ridge, 
function values are -0.990283. It is easy to converge to local optimal global solution near the lows, but difficult to converge to the global optimal solution.

We make the termination of SGA and IGA algorithm in 100 generations. We can see minimal algebraic convergence, convergence in probability, and mean convergence value and convergence standard deviation of two algorithms after 100 random simulation in Table 1.

Table 1 Standard genetic algorithm and adaptive genetic algorithm improving the convergence performance comparison

\begin{tabular}{cccccccc}
\hline Function & Algorithm & $\begin{array}{c}\text { The } \\
\text { solution } \\
\text { population } \\
\text { size }\end{array}$ & $\begin{array}{c}\text { Algorithm } \\
\text { to } \\
\text { terminate } \\
\text { algebra }\end{array}$ & $\begin{array}{c}\text { Minimum } \\
\text { convergence } \\
\text { algebra }\end{array}$ & $\begin{array}{c}\text { Convergent } \\
\text { probability }\end{array}$ & $\begin{array}{c}\text { The average } \\
\text { value of } \\
\text { convergence }\end{array}$ & $\begin{array}{c}\text { The standard } \\
\text { deviation of } \\
\text { convergence }\end{array}$ \\
\hline \multirow{2}{*}{$f_{1}$} & SGA & 100 & 100 & 40 & 52 & -0.99595 & 0.00091 \\
\hline
\end{tabular}

As can be seen from the table 1, IGA's minimum convergence algebra, convergence probability, convergence standard deviation, the convergence of the average value is better than SGA. This means that the convergence speed and convergence accuracy of IGA is better than that of SGA. IGA has a better function.

Through the simulation of complex function, the results show that the improved genetic algorithm is more effective to achieve global optimization, promote the efficiency of evolution, and can solve the complex optimization problems with strong stability.

\section{A global optimization method for the minimum safety factor of rock soil slope based on genetic algorithm}

Process for rock and soil slope stability analysis can be summarized into four steps: a. give a sliding plane was determined; b. of slip surface shear compressive static analysis; c. calculate the slope safety coefficient $\mathrm{k}$; $\mathrm{d}$. use searching algorithm to determine the slope of the minimum safety factor $k_{\min }$ and the corresponding critical slip surface.

If we use the traditional nonlinear optimization method for slope stability analysis, since the conventional optimization method has the disadvantage of a non-robust, more numerical solution would be given and it's easy to search for a local minimum factor of safety solutions rather than the global minimum. The optimization method based on genetic algorithms genetics through continuous evolution can search the global optimal solution, this algorithm has a strong parallel global search capability. In this paper, the improved algorithm can improve the convergence, to avoid local optima. With improved genetic algorithm, minimal safety factor Solution. Improved genetic algorithm in the whole process of evolution, only the fitness function as the basis, without using any external information, the search is based on the fitness value of each individual group. Therefore the choice of fitness function, the convergence rate of the algorithm, as well as its global search capability have an important impact. Groups of individual i fitness function is defined by the following formula:

$$
f_{i}=C-k_{i}
$$

In this formula, $f_{i}$ represents the i-th group of individual fitness function value. $\mathrm{C}$ is constant. The fitness function value of the individual must be positive in the whole process of evolution. ${ }_{i}$ indicates the objective function value of the first individual.

We use the genetic algorithm searches the minimum safety factor of slope. The operation is carried out under a binary, so we need binary and decimal (real) encoding conversion. The formula of binary string converted to decimal conversion for Interval [ $\left.k_{i \text { min }}, k_{\text {iman }}\right]$ is: 


$$
k_{i}=k_{i \text { min }}+\left(k_{\text {iman }}-k_{x \text { min }}\right) \sum_{a=0}^{Q} g_{a} 2^{a} /\left(2^{Q}-1\right)
$$

In this formula, $k_{i \text { min }}, k_{\text {iman }}$ determine the slope of the search range, the minimum and maximum safety factor according to prior information. Q represents the code length, $g_{a}$ represents the first binary encoding gene a-th.

\section{Nanning levee typical slope failure mechanism research based on the inversion analysis}

\section{Nanning flood project overview and typical slope selection}

Nanning Ximing River, a total length of $6.060 \mathrm{~km}$, which the bank downstream begins to the Xi Xiangtang area and upstream end stop at Shibu Ling east.. The project in a design flood control standard for fifty years, has a crest elevation of $82.40 \mathrm{~m} \sim 82.69 \mathrm{~m}$ and a $5 \sim 8 \mathrm{~m}$ high embankment, the largest bank of the gully up to $20 \mathrm{~m}$, embankment design using an earth embankment. Ximing embankment located on the left bank Yongjiang leading edge in grade II terrace, flat ground and the ground elevation is $72.4 \mathrm{~m} \sim 78.5 \mathrm{~m}$. its embankment go to straight and the upstream section of the river bend trend to be a concave bank segment, generally owning $20^{\circ} \sim 50^{\circ}$ in a steeper slope and stability is poorer, The slope groundwater depth is $1 \sim 8 \mathrm{~m}$, which belong to a weak lithology layer. The inner factor of bank slope instability is that the drainage difficulty of ground water in flooding process and resulting that the buried depth of ground water makes soil saturation state for a long time. According to a bank material internal influence and the erosion of bank determination, the entire length of the bank slope are belong to the bank slope instability in the flood period. Inside and outside the embankment slope is $1: 2$, 1: 1.5, filling levee complex, poor quality embankment, no levee foundation seepage control measures, in order to test the river bank at the time of design flood water level dips stability of the river bank, the paper select $2+991$ slope section of Nanning Ximing Embankment analysis.

\section{Mechanism of flood damage based on inversion analysis of geotechnical parameters}

\section{Analysis of dike slope based on genetic algorithm inversion analysis}

We make the improved genetic algorithm inversion method and finite difference software FLAC3D $[5,6]$ combined to find a different slope corresponding to the height of the water level drops factor of safety and stability in fifty years of design flood conditions. We made the field slope geological data collected as the basis. We searched out the safety factor is 1.00 , corresponding drawdown of critical height $\mathrm{H}$, and analyzed the mechanism of landslide damage.

\section{Mechanical parameters of slope of levees}

Each section of soil properties of typical parameters is in Table 2. The crest width is $8 \mathrm{~m}$, inside and outside the embankment slope is $1: 1.5$ to $1: 2$.

Table 2 Numerical simulation of mechanical parameters

\begin{tabular}{|c|c|c|c|c|c|c|c|c|c|}
\hline No. & $\begin{array}{l}\text { Soil } \\
\text { types }\end{array}$ & $\begin{array}{c}\text { Natural } \\
\text { unit } \\
\text { weight } \\
\left(\mathrm{kN} / \mathrm{m}^{3}\right)\end{array}$ & $\begin{array}{c}\text { Saturated } \\
\text { unit } \\
\text { weight } \\
\left(\mathrm{kN} / \mathrm{m}^{3}\right)\end{array}$ & $\begin{array}{l}\text { Modulus } \\
\text { of } \\
\text { elasticity } \\
\text { (MPa) }\end{array}$ & $\begin{array}{l}\text { Poisson } \\
\text { ratio }\end{array}$ & $\begin{array}{c}\text { Shear } \\
\text { strength } \\
(\mathrm{kPa})\end{array}$ & $\begin{array}{c}\text { Cohesion } \\
(\mathrm{kPa})\end{array}$ & $\begin{array}{c}\text { Internal } \\
\text { friction } \\
\text { angle } \\
(\boldsymbol{\Phi})\end{array}$ & $\begin{array}{c}\text { Osmotic } \\
\text { coefficient } \\
(\mathrm{cm} / \mathrm{s})\end{array}$ \\
\hline 1 & Clay & 19.0 & 20.1 & 4.6 & 0.35 & 79.4 & 15 & 12 & $1 * 10^{-3}$ \\
\hline 2 & $\begin{array}{l}\text { Silty } \\
\text { clay }\end{array}$ & 19.9 & 20.9 & 4.5 & 0.28 & 35.1 & 35 & 10 & $1 * 10^{-6}$ \\
\hline 3 & Silt & 19.7 & 20.5 & 5.7 & 0.26 & 107.2 & 18 & 6 & $1 * 10^{-4}$ \\
\hline 4 & $\begin{array}{c}\text { Mealy } \\
\text { sand }\end{array}$ & 19.7 & 20.3 & 8.9 & 0.22 & 165.2 & 10 & 8 & $1 * 10^{-3}$ \\
\hline 5 & $\begin{array}{c}\text { Round } \\
\text { sand }\end{array}$ & 19.8 & 19.8 & 16 & 0.20 & 213 & 0 & 30 & $1 * 10^{-2}$ \\
\hline
\end{tabular}




\section{inversion analysis calculation step}

(1) According to the site to collect slope geological data, to determine the inversion of levee slope fifty years of design flood conditions, the water level drop height range $\mathrm{H}=\left\{0, \mathrm{H}_{\max }\right\}$.

(2) Randomly generated initial population, $M_{i}=M(t)$, using an iterative method to calculate the slope of levees in different time periods, different drawdown safety factor corresponding to the height $\mathrm{k}$.

(3) In this paper, the use of genetic manipulation improved crossover and mutation, and global search strategy evolutionary step.

(4) The implementation of the convergence conditions, when the condition is satisfied, the operation ends, and the resulting solution search space is converted to solution of the problem. Otherwise, it returns the third step, until convergence conditions are satisfied so far.

Because of different solutions in different inversion model, select the appropriate objective function and inverse mathematical model is very important, when the slope before the water level drops to a certain height range, are equipped to meet the stability factor $\mathrm{k}=1.00$ embankment slope conditions but only one set of mechanical parameters meet the stability factor $\mathrm{k}=1.0$, as well as the calculated slope geotechnical parameters close to the actual, therefore, based on the levees slope field geological survey data, the establishment of genetic algorithm based on improved inversion. Numerical modeling of the slope parameter of the method is conducive to solving the embankment slope geotechnical parameters and engineering phase close.

\section{The inversion calculation model}

A numerical model[$[7,8]$ need to be established in application of finite difference method to analyze levees slope's seepage field and stability. Since most of the levees slope is made of soil, during the stability analysis, it is assumed as a three-dimensional solid structure. With the slope water level drops, when seepage is analyzed, different parameter values above and below wetting line need to be considered, the change in position need to be considered as well. The establishment of model grid follows the modes of sub-regional modeling. FISH language is used to adjust basic grid nodes to generate 3D mesh models. As long as the sliding surface boundaries within the scope of the border, chosen of the model boundaries has little effect to the strength reduction results. Therefore the proposed model takes the distance from foot of the slope to the left margin equal to $1.45 \mathrm{H}(\mathrm{H}$ is the height of levees slope), the distance from the top of the hill to the right margin equal to $2.4 \mathrm{H}$ and from top of the hill to the bottom border $2 \mathrm{H}$ distance equal manner to expand. Since the strength reduction is based on numerical simulation and numerical simulation technique relies on the grid, the calculation accuracy of the results will be influenced by grid density. The number of FLAC3D grid are very sensitive, so if the grid is too dense, the calculation takes too long time. Therefore, according to the analysis of actual needs, choose the right mesh density for analysis, levee slope model in this paper is divided into a total of 28828 grid cells, node 4330.

\section{Inversion numerical results and analysis}

\section{Numerical outcomes}

(1) Numerical analysis of water level which drops from design flood water level but does not reach the critical depth

With improved genetic algorithm inversion method, it's found out that when water level drops from design flood water level but does not reach to the critical depth, different drop height corresponds to different safety factor of embankment slope. When the drop height is selected of $2 \mathrm{~m}, 4 \mathrm{~m}$, and $5 \mathrm{~m}$, safety factor model is 1.15,1.05 and 1.02. Levees slope sliding failure does not occur, but potentially dangerous slip surface exists in the embankment slope.

(2) Numerical analysis of water level which drops from design flood water level to the critical depth

When the water level drops $5.5 \mathrm{~m}$, safety factor of dike slope is 1.00 , in which case the levee slope is in a critical state. Water level drawdown has a certain effect to local stress distribution of slope model. It is because the water level drops, so that the original stability mechanical of slope changes. 
When the water level drops to $5.5 \mathrm{~m}$, significant changes have occurred in displacement and velocity field. The maximum displacement and velocity all concentrate in the top of the hill slope and corners. Slip surface run through in the slope area. So it's thought that slope is at a instability state.

\section{Water level test of calculation results}

Using the traditional limit equilibrium method, the results of FLAC3D strength reduction is checked and verified. Swedish slice method is one of limit equilibrium method, considering penetration force, irrespective of the outer slope of the hydrostatic pressure, without regard to earthquakes. When gravity tangential component of soil slice is against sliding force direction, it's only treated as sliding force. Then slope model stability calculation is made within design flood water level drawdown conditions. Slice method of soil stripe width is $2 \mathrm{~m}$. When searching most dangerous slip surface, the center step takes $2 \mathrm{~m}$, searching radius step takes $2 \mathrm{~m}$. It's searched out that the most unfavorable sliding surface sliding radius is $\mathrm{R}=35.086 \mathrm{~m}$.

When the water level drops $5.5 \mathrm{~m}$ results when strength reduction factor of safety is 1.0 , while the results of the safety factor limit equilibrium method is 1.01. Comparison of the results combined with improved genetic algorithm inversion method FLAC3D strength reduction and limit equilibrium method can be seen that the calculation of design flood water level dropped to $5.5 \mathrm{~m}$ height, the most dangerous sliding surface and the safety factor obtained by the two methods. It's indicated that the improved genetic algorithm inversion and simulation software FLAC3D combined for solving the slope safety factor and the search for the most dangerous slip surface is feasible and effective.

\section{Conclusion}

(1) Genetic algorithm has better global search capability than traditional optimization methods for solving complex issues and complex objective function objective function, more advantages, easier to use. But when GA is used to solve some practical problems, it usually converge to a local solution, rather than globally optimal solution. Based on the standard genetic algorithm crossover and mutation operation two genetic improvements, improved Genetic algorithm has better global search capability.

(2)Improved genetic algorithm inversion method and numerical software FLAC3D is combined to analyze typical flood slope. At the design flood conditions, dike safety factor corresponding to different drawdown height is got in this paper. When embankment slope safety factor $\mathrm{k}=1.00$, drawdown height is $5.5 \mathrm{~m}$. And the slope slip surface is found.

(3) For complex geotechnical layer slope, limit equilibrium method is done to simplify the calculation, to get analytical solutions based on Striped force hypothesis. Strength reduction calculated safety factor is based on the stress - strain analysis results, with no need of striped force hypothesis which considers the effect of the stress distribution in rock and soil so that numerical method calculation is more accurate, numerical simulation is closer to the actual situation of rock and soil slope. The calculation speed of the search is also faster, calculation method is more convenient than limit equilibrium method, and has a higher applicability.

\section{Acknowledgements}

This work was financially supported by National Natural Science Foundation of China (51369005), Systemic research project of Guangxi key laboratory of Disaster Prevention and Engineering Safety, Guangxi University, Nanning, China (2013ZDX04).

\section{References}

[1] Chongwu Ma, Zhongyu Liu, Tiande Miao, Dekai Wang. River water level on the embankment slope stability [J] (In Chinese). Lanzhou University, 2000,36 (3): $56 \sim 60$. 
[2] Xinxi Liu, Yuanyou Xia, Xianshu Zhang, Ruiqing Guo. Water level drop impact on the stability of the landslide [J] (In Chinese). Journal of geotechnical mechanics and engineering, 2005, 24 (8) : $1339 \sim 1444$.

[3] Haiwei Yang, Yong Feng. Water level drop impact on the stability of reservoir bank landslide [J] (In Chinese). Journal of railway engineering, 2007, 8:74 76.

[4] Weimin Shi, Yingren Zheng. Water level decline situation of landslide stability analysis [J] (In Chinese), Journal of water conservancy, 2004, (3) : $76 \sim 80$.

[5] Honglei Lin, Yuan Ou. Analysis of slope stability based on FLAC3D strength subtraction [J] (In Chinese), Geotechnical engineering and underground engineering, 2010, 30 (1) : $87 \sim 88$.

[6] Lipeng Liu, Qi Chen, Bin Zhang. Slope stability Study based on FLAC strength reduction theory [J] (In Chinese), Geotechnical engineering, 2008, 22 (1) : $6 \sim 10$.

[7] Cong Ji, Lei Nai, Hong Ma, Lujun Liu. Application of FLAC3D strength reduction theory in slope stability analysis[J] (In Chinese). Global Geology. 2013,32 (1) : 158-164.

[8] Xuezhi Wang, Zhai Cheng, Chong Bi, Chaoyan Zhu. FLAC3D application in slope stability analysis [J] (In Chinese). Journal of JiLin water conservancy, 2010, 6:39-41.

\section{Bibliography}

Rongyong Ma (1955- ), male, the Zhuang nationality, professor, Nanning, China. E-mail: mry57@gxu.edu.cn. 